\title{
SOJOURN TIME DISTRIBUTIONS IN A MARKOVIAN G-QUEUE WITH BATCH ARRIVAL AND BATCH REMOVAL
}

\author{
YANG WOO SHIN \\ Changwon National University \\ Department of Statistics \\ 9 Sarim-dong, Changwon, Kyung-nam, 641-773, Korea \\ e-mail:ywshin@sarim.changwon.ac.kr
}

(Received November, 1997; Revised January, 1999)

\begin{abstract}
We consider a single server Markovian queue with two types of customers; positive and negative, where positive customers arrive in batches and arrivals of negative customers remove positive customers in batches. Only positive customers form a queue and negative customers just reduce the system congestion by removing positive ones upon their arrivals. We derive the LSTs of sojourn time distributions for a single server Markovian queue with positive customers and negative customers by using the first passage time arguments for Markov chains.
\end{abstract}

Key words: Negative Customers, First Passage Times, Batch Arrival, Batch Removal, Sojourn Times.

AMS subject classifications: $60 \mathrm{~K} 25$.

\section{Introduction}

We consider a queue with two types of customers; positive and negative. Positive customers are ordinary ones who, upon arrival, join the queue with the intention of being served. In contrast to the positive customers, the arrival of a negative customer removes some of the positive customers from the system, if any available, and then disappears; otherwise the negative customer is lost. Only positive customers can form a queue and negative customers just reduce system congestion. Such queues have been called G-queue (Harrison and Pitel [12]).

Since Gelenbe [7] introduced the notion of negative customers to represent the inhibitator signal in neural networks and commands to delete some transactions in distributed computer systems or databases, there has been a growing interest not only in networks of queues $([3-9,12,14,15])$ but also in single node queues with negative customers $([1,2,10,11,13,16])$. Interest in time delays in the $G$-queue has increased recently. Harrison and Pitel [11] derived the LSTs of the sojourn time distributions for 
the $\mathrm{M} / \mathrm{M} / 1 G$-queue under the combinations of various queueing disciplines and removal strategies. Harrison and Pitel [12] investigated the end-to-end delay in an open tandem pair of a $G$-queue with FCFS discipline and RCE removal strategy. Most papers assume that upon arrival to a queue, a negative customer removes an ordinary customer from the queue. Recently, several authors have generalized this concept, allowing a negative arrival to remove a batch of customers (Henderson et al. [15], Chao and Pinedo [5] and Gelenbe [8]), a random amount of workload (Boucherie and Boxma [2]), or even all work in the system (Chao [4] and Jain and Sigman [16]).

However, the results about sojourn time distribution even for single node $G$-queues with batch arrival or batch removal are few to the author's best knowledge. In this paper, we use the first passage time arguments of Markov chains to derive the LST of the sojourn time distribution in single server Markovian $G$-queues with a batch arrival of positive customers and/or batch removal by a negative arrival. The mathematical accessibility of our model compared with that of Harrison and Pitel [11] represents a part of the motivation for the study of batch arrivals/ removals. Furthermore, our model is related to the inventory systems with perishable products such as fruit, vegetables, and meat, in which arrival and removal occur in batches and instantaneous removal of inventory usually depends on the length of time that the products spent in the system.

This paper is organized as follows. We describe the model in detail and derive the queue length distribution in equilibrium in Section 2. In Section 3, we derive the LSTs of the first passage times related to the compound Poisson processes. The LSTs of sojourn time distributions, under combinations of two queue disciplines, FCFS and LCFS and two removal strategies, RCE and RCH are derived in Sections 4-7.

\section{Queue Length Distribution}

In this section, we describe the mathematical model in detail and derive the queue length distribution in equilibrium at the arrival instants of positive customers. We consider a single server queue in which positive customers arrive in batches according to a Poisson process with rate $\lambda^{+}$, and negative customers arrive according to Poisson process with rate $\lambda^{-}$, which is independent of the arrival process of positive customers. We assume that each arrival of a negative customer removes a random number $B$ of positive customers in the system. That is, upon a negative arrival, if there are $k$ positive customers in the system, $\min (B, k)$ positive customers are removed and the negative customer disappears. The service time distribution of all customers is exponential with mean $\frac{1}{\mu}$. For the notational simplicity, we let $\tilde{\mu}=\lambda^{-}+\mu$ and $\lambda=\lambda^{+}+\lambda^{-}$. We assume that the batch size $A$ of positive customers and the quota $B$ of a negative customer take finite values to avoid calculations of infinite matrices. However, this assumption is not a strong restriction, since the supports of $A$ and $B$ may be arbitrarily large and one can apply our model to $A$ and $B$ taking infinite values by truncating the tail parts of the state spaces with sufficiently small tail probabilities. Let $P(A=n)=a_{n}$ and $P(B=n)=b_{n}, \quad n=1,2, \ldots$ with $a_{n}=0$, $n \geq l+1$ and $b_{n}=0, n \geq m+1$ for some $1 \leq l, m<\infty$. We denote the means $\bar{a}=E(A)$ and $\frac{n}{b}=E(B)$ and generating functions $A(z)=\sum_{n=1}^{l} a_{n} z^{n}$ and $B(z)$ $=\sum_{n=1}^{m} b_{n} z^{n}$.

Note that the stationary distribution of the queue length in this system is invari- 
ant under the service discipline and removal strategies and concern only positive customers. This model is equivalent to the $\mathrm{M}^{A} / \mathrm{M}^{B} / 1$ queue where customers arrive in batches with batch size $A$ according to a Poisson process with rate $\lambda^{+}$and the customers are served in batches of maximum size $\widetilde{B}$ with $\widetilde{b}_{k}=P(\widetilde{B}=k), 1 \leq k \leq m$, where

$$
\tilde{b}_{k}=\left\{\begin{array}{cc}
\frac{\lambda-b_{1}+\mu}{\widetilde{\mu}}, & k=1 \\
\frac{\lambda^{-} b_{n}}{\tilde{\mu}}, & 2 \leq k \leq m,
\end{array}\right.
$$

and the service time distribution is exponential with parameter $\tilde{\mu}$. The necessary and sufficient condition for this system to be positive recurrent is given (e.g. Miller [17]) by

$$
\rho=\frac{\lambda^{+} \bar{a}}{\mu+\lambda^{-} \bar{b}}<1
$$

We assume that $\rho<1$ throughout.

Now we turn our attention to the queue length distribution at the arrival epochs of positive customers, which will be imperative in the upcoming sections. Let $\left\{N_{n}\right\}$ be the number of positive customers in the system at the epoch immediately before the arrival of the $n$th batch of positive customers. Let $A_{n}$ be the batch size of the $n$th arrival of positive customers with the same distributions as $A$ and $D_{n+1}$, where $D_{n+1}$ is the number of positive customers departed from the system during the $(n+1)$ st interarrival period of the batch of positive customers. Then it can be seen that

$$
N_{n+1}=\max \left(N_{n}+A_{n}-D_{n+1}, 0\right)
$$

The probability $d_{n}$ that $n$ positive customers potentially leave the system during the interarrival time of a batch of positive customers is given by

$$
d_{n}=\left\{\begin{array}{cc}
p, & n=0 \\
\sum_{j=1}^{n} b(j, n) p q^{j}, & n \geq 1
\end{array}\right.
$$

where $p=\frac{\lambda^{+}}{\lambda^{+}+\bar{\mu}}, q=1-p$ and $b(j, n)$ is the $j$-fold convolution of the probability mass function $\left\{\tilde{b}_{k}, 0 \leq k \leq m\right\}$. Simple calculations yield

$$
\widetilde{B}(z)=\sum_{n=1}^{m} \widetilde{b}_{n} z^{n}=\frac{1}{\widetilde{\mu}}\left(\mu z+\lambda^{-} B(z)\right),
$$

and hence the probability generating function $d(z)=\sum_{n=0}^{\infty} d_{n} z^{n}$ is given by

$$
d(z)=\frac{\lambda^{+}}{\lambda^{+}+\mu(1-z)+\lambda^{-}(1-B(z))}
$$

Denoting $d_{n}=0$ for $n \leq-1$ and $\bar{d}_{n}=\sum_{k=n}^{\infty} d_{k}, n \geq 0$, we deduce that the transition probability matrix $P=\left(p_{i j}\right)$ of $\left\{N_{n}\right\}$ is given by 


$$
p_{i j}=\left\{\begin{array}{cc}
\sum_{k=1}^{l} a_{k} \bar{d}_{i+k}, & \text { if } j=0 \\
\sum_{k=1}^{l} a_{k} d_{k+i-j}, & \text { if } 1 \leq j \leq i+l \\
0, & \text { if } j \geq i+l+1 .
\end{array}\right.
$$

Following similar procedures as those in Miller [17], the stationary distribution $\pi=$ $\left\{\pi_{i}, i=0,1, \ldots\right\}$ of $\left\{N_{n}\right\}$ is given by

$$
\pi_{k}=C \sum_{i=1}^{K} \sum_{j=0}^{n_{i}-1} c_{i j}\left(\left.\frac{d^{j}}{d x^{j}} x^{k}\right|_{x=\alpha_{i}}\right), k \geq 0
$$

where $\alpha_{i}, 1 \leq i \leq K$, is the solution of the equation

$$
\left.\alpha^{l}=d(\alpha) a_{1} \alpha^{l-1}+a_{2} \alpha^{l-2}+\ldots+a_{l}\right)
$$

with $n_{i}$ being the multiplicity of $\alpha_{i}(1 \leq i \leq K)$, such that $1 \leq n_{i} \leq l$ and $\sum_{i=1}^{K} n_{i}=l . \quad c_{i j}, 0 \leq j \leq n_{i}-1,1 \leq i \leq K$ are arbitrary constants, which can be determined by the $l-1$ simultaneous equations:

$$
\pi_{j}=\sum_{i=0}^{\infty} \pi_{i} p_{i j}, \quad j=1,2, \ldots, l-1
$$

under the constraint

$$
\sum_{i=1}^{K} \sum_{j=0}^{n_{i}-1} c_{i j}=1
$$

and $C$, the normalizing constant (in $\sum_{i=0}^{\infty} \pi_{i}=1$ ), is given by

$$
C=\left[\sum_{i=1}^{K} \frac{c_{i 0}}{1-\alpha_{i}}+\sum_{i=1}^{K} \sum_{j=1}^{n_{i}-1} c_{i j} \frac{j !}{\left(1-\alpha_{i}\right)^{j+1}}\right]^{-1}
$$

After simple but tedious algebra, we have from (2.3) and (2.4) the following linear system of equations for $\left\{c_{i j}, 1 \leq i \leq K, 0 \leq j \leq n_{i}-1\right\}$ :

where

$$
H c=e_{l},
$$

$$
\boldsymbol{c}=\left(c_{10}, c_{11}, \ldots, c_{1, n_{1}-1}, c_{2,0}, c_{2,1}, \ldots, c_{2, n_{2}-1}, \ldots, c_{K, n_{K}-1}\right)^{t}
$$

$e_{l}=(0,0, \ldots, 0,1)^{t}$ is the $l$-unit vector and $H$ is the $l \times l$ matrix with its $k$ th $(1 \leq k \leq l-1)$ row

$$
\boldsymbol{h}_{k}=\left(h_{10}(k), h_{11}(k), \ldots, h_{1, n_{1}-1}(k), h_{2,0}(k), \ldots, h_{2, n_{2}-1}(k), \ldots, h_{K, n_{K}-1}(k)\right.
$$

and $l$ th row $h_{l}=(1,1, \ldots, 1)$, and for $1 \leq k \leq l-1,1 \leq i \leq K, 0=j \leq n_{i}-1$,

$$
h_{i j}(k)=\sum_{r=k+1}^{l} a_{r} \sum_{n=0}^{r-k-1}(k-r+n)(k-r+n-1) \ldots(k-r+n-j+1) \alpha_{i}^{k-r+n-j} .
$$

\section{Special Cases}

(1) Let $l=1$, that is, $A \equiv 1$.

In this case, $(2.2)$ becomes $\tilde{\mu} \alpha \widetilde{B}(\alpha)-\left(\lambda^{+}-\tilde{\mu}\right) \alpha+\lambda^{+}=0$ and it has a unique 
solution in $0<\alpha<1$, say $\alpha_{0}$, and the stationary distribution is given by

$$
\pi_{n}=\left(1-\alpha_{0}\right) \alpha_{0}^{n}, n \geq 0 .
$$

(2) Let $l=1$ and $m=1$, that is, $A \equiv 1$ and $B \equiv 1$.

In this case, $(2.2)$ becomes $\alpha^{2}-(1+\rho) \alpha+\rho=0$ and the stationary distribution is given by

$$
\pi_{n}=(1-\rho) \rho^{n}, n \geq 0 \text {. }
$$

\section{The First Passage Times}

The sojourn times, which will be treated in the upcoming sections, can be considered as the first passage times of the corresponding Markov chains. So we need to investigate the first passage times for some Markov chains related to compound Poisson processes.

First, we consider the compound Poisson process

$$
X(t)=\sum_{i=1}^{N(t)} X_{i}
$$

where $\{N(t), t \geq 0\}$ is a Poisson process with rate $\nu$ and $\left\{X_{i}\right\}$ is a sequence of independent and identically distributed (i.i.d.) random variables, which are independent of $\{N(t), t \geq 0\}$ and have probability mass function $x_{k}=P\left(X_{1}=k\right), k=1,2, \ldots$ and probability generating function $\Xi(z)=\sum_{n=1}^{\infty} x_{n} z^{n},|z| \leq 1$. Let $U_{X}(n)$ be the first passage of time of $X(t)$ to the state $n$, that is

$$
U_{X}(n)=\inf \{t \geq 0: X(t) \geq n\}
$$

and let $U_{X}(n, t)=P\left(U_{X}(n) \leq t\right)$ be the probability distribution function of $U_{X}(N)$. By conditioning the first transition of the process $\{X(t)\}$, we have the following proposition.

Proposition 1: The LST $U_{X}^{*}(n, s)$ of $U_{X}(n, t)$ is given recursively by

$$
\begin{gathered}
U_{X}^{*}(1, s)=\frac{\nu}{\nu+s} \\
U_{X}^{*}(n, s)=\frac{\nu}{\nu+s}\left(\bar{x}_{n}+\sum_{i=1}^{n-1} x_{i} U_{X}^{*}(n-i, s)\right), n \geq 2
\end{gathered}
$$

where $\bar{x}_{i}=\sum_{k=i}^{\infty} x_{k}, i \geq 1$. The double transform $\tilde{U}_{X}^{*}(z, s)=\sum_{n=1}^{\infty} z^{n} U_{X}^{*}(n, s)$ is given by

$$
\tilde{U}_{X}^{*}(z, s)=\frac{z}{1-z} \frac{\nu(1-\Xi(z))}{s+\nu(1-\Xi(z))} .
$$

Now we consider the difference of two independent compound Poisson processes

$$
X_{1}(t)=\sum_{i=1}^{N_{1}(t)} X_{1, i} \text { and } X_{2}(t)=\sum_{i=1}^{N_{2}(t)} X_{2, i}
$$

where $\left\{N_{1}(t)\right\}$ and $\left\{N_{2}(t)\right\}$ are independent Poisson processes with rates $\lambda_{1}$ and $\lambda_{2}$, respectively, and $\left\{X_{1, i}\right\}$ and $\left\{X_{2, i}\right\}$ are independent sequences of i.i.d. random variables with $P\left(X_{1, i}=k\right)=x_{1, k}, \quad k \geq 1$ and $P\left(X_{2, i}=k\right)=x_{2, k}, \quad 1 \leq k \leq m$. We assume that the random variable $X_{2, i}$ is bound by $m$. Define a Markov chain 


$$
Z(t)=X_{1}(t)-X_{2}(t), t \geq 0
$$

with $Z(0)=0$. Let $Z_{n}$ be the state at the instant immediately after the $n$th transition of the process $\{Z(t), t \geq 0\}$ and $\tau_{n}$ the time interval between the $n$th and $n+1$ st transitions. Then $\left\{\left(Z_{n}, \tau_{n}\right), n \geq 0\right\}$ is a Markov renewal process with the transition probability matrix $Q_{Z}(t)$ of the form

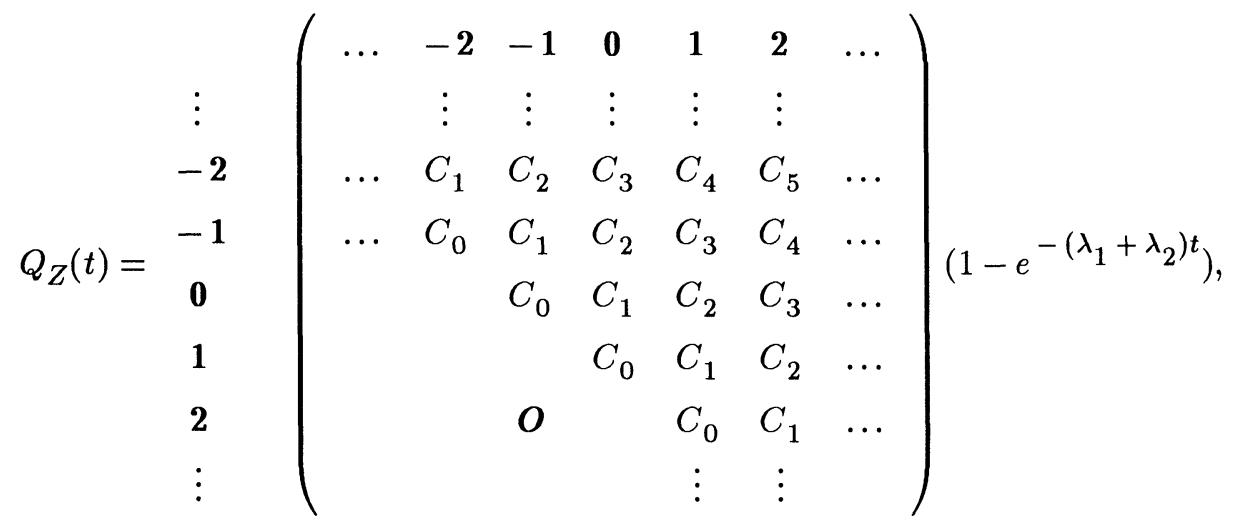

where each level $\boldsymbol{i}=((i, 1),(1,2), \ldots,(i, m)), i=0, \pm 1, \pm 2, \ldots$ is the set of $m$ states, the state $(i, k)$ in level $i$ means the state $(i, k)=m i+k-1, C_{0}$ is the upper triangular matrix

$$
C_{0}=\left(\begin{array}{ccccc}
c_{-m} & c_{-m+1} & c_{-m+2} & \cdots & c_{-1} \\
& c_{-m} & c_{-m+1} & \cdots & c_{-2} \\
& & c_{-m} & \cdots & c_{-3} \\
& O & & \ddots & \vdots \\
& & & & c_{-m}
\end{array}\right)
$$

and

$$
C_{n+1}=\left(\begin{array}{cccc}
c_{m n} & c_{m n+1} & \ldots & c_{m n+m-1} \\
c_{m n-1} & c_{m n} & \ldots & c_{m n+m-2} \\
\vdots & \vdots & & \vdots \\
c_{m n-m+1} & c_{m n-m+2} & \cdots & c_{m n}
\end{array}\right), n \geq 0
$$

where

$$
c_{i}=\left\{\begin{array}{cc}
\frac{\lambda_{1}}{\lambda_{1}+\lambda_{2}} x_{1, i} & \text { if } i \geq 1 \\
\frac{\lambda_{2}}{\lambda_{1}+\lambda_{2}} x_{2,-i} & \text { if }-m \leq i \leq-1 \\
0 & \text { if } i=0 .
\end{array}\right.
$$

Define the first passage time as 


$$
G_{Z}(n)=\inf \{t \geq 0: Z(t) \leq n\}
$$

and denote its distribution function by $G_{Z}(n, t)=P\left(G_{Z}(n) \leq t\right)$. Now we derive the LST $G_{Z}^{*}(-n, s)$ of $G_{Z}(-n, t), n \geq 1$.

Proposition 2: The LSTs $G_{Z}^{*}(-n, s)$ for $1 \leq k \leq m$, are recursively given by

$$
\begin{gathered}
G_{Z}^{*}(-1, s)=\sum_{j=1}^{m}\left[H^{*}(s)\right]_{1 j}, \\
G_{Z}^{*}(-k, s)=\sum_{j=1}^{m-k+1}\left[H^{*}(s)\right]_{\substack{j \\
j=m-k+2}}+\sum_{\substack{m \\
j=}}^{m}\left[H^{*}(s)\right]_{1 j} G_{Z}^{*}(m-k+1-j, s),
\end{gathered}
$$

and for $n \geq 2$ and $k=m, m-1, \ldots, 1$, by

$$
G_{Z}^{*}(-m n+k-1, s)=\sum_{j=1}^{k}\left[\left(H^{*}(s)\right)^{n}\right]_{1 j}+\sum_{j=k+1}^{m}\left[\left(H^{*}(s)\right)^{n}\right]_{1 j} G_{Z}^{*}(k-j, s),
$$

where $H^{*}(s)$ is an $m \times m$ matrix, which is the minimal nonnegative solution of the matrix equation

$$
H^{*}(s)=\left(\frac{\lambda_{1}+\lambda_{2}}{\lambda_{1}+\lambda_{2}+s}\right) \sum_{n=0}^{\infty} C_{n}\left[H^{*}(s)\right]^{n}
$$

while $\left[H^{*}(s)\right]_{i j}$ denotes the $(i, j)$ entry of the matrix $H^{*}(s)$.

Proof: Let $T\left(i+r, j ; i, j^{\prime}\right)$ be the first hitting time of $\{Z(t), t \geq 0\}$ from state $(i+r, j)=m(i+r)+j-1, r \geq 1,1 \leq j \leq m$, to state $\left(i, j^{\prime}\right)=m i+j^{\prime}-1,1 \leq j^{\prime} \leq$ $m$, with the additional requirement that $\left(i, j^{\prime}\right)$ is the first state at level $i$ to be visited and $\tau_{\boldsymbol{i}}(j, k)$ is the first passage time from state $(i, j)$ to state $(i, k), 1 \leq j, k \leq m$, $j-k>0$. When the process $\left\{Z_{n}\right\}$, starting at $(0,1)$, that is, $Z_{0}=0$, hits the level $-\boldsymbol{n}$, and visits state $(-n, j) \in\{(-n, 1), \ldots,(-n, k)\}$, then $G_{Z}(-m n+k-1)=$ $T(0,1 ;-n, j)$; and if the state visited is $(-n, j) \in\{(-n, k+1), \ldots,(-n, m)\}$, then $G_{Z}(-m n+k-1)$ is the sum of $T(0,1 ;-n, j)$ and $\tau_{-n}(j, k)$. Thus we have for $n \geq 1,1 \leq k \leq m$,

$$
\begin{gathered}
P\left(G_{Z}(m n-k+1) \leq t\right)=\sum_{j=1}^{k} P(T(0,1 ;-n, j) \leq t) \\
\quad+\sum_{j=k+1}^{m} P\left(T(0,1 ;-n, j)+\tau_{-n}(j, k) \leq t\right) .
\end{gathered}
$$

Let $H_{j j^{\prime}}^{[r]}(t)=P\left(T\left(i+r, j ; i, j^{\prime}\right) \leq t\right)$ be the distribution function of $T\left(i+r, j ; i, j^{\prime}\right)$ and $H_{j j^{\prime}}^{[r] *}(s)$ be the LST of $H_{j j^{\prime}}^{[r]}(t), 1 \leq j, j^{\prime} \leq m$. Let $H^{[r]}(t)$ and $H^{[r] *}(s)$ denote the $m \times m$ matrices with $\left(j, j^{\prime}\right)$ entry $H_{j j^{\prime}}^{[r]}(t)$ and $H_{j j^{\prime}}^{[r] *}(s)$, respectively. By the spatial homogenuity for levels of $Q_{Z}(t)$ the distribution of $T\left(i+r, j ; i, j^{\prime}\right)$ does not depend on level $i$ but only on $r$ and $\left(j, j^{\prime}\right)$ and hence we get

$$
H^{[r] *}(s)=\left[H^{*}(s)\right]^{r}, \quad r \geq 1 .
$$


From the spatial homogeneity of the transition probability $Q_{Z}(t)$ for states $\tau_{\boldsymbol{i}}(j, k)$, $j>k$, depends only on the difference of the states $j-k$ and its distribution function is the same as that the $G_{Z}(k-j)$. Note that, by the Markovian property, $T(0,1 ;-n, j)$ and $\tau_{-n}(j, k), n \geq 1$ are independent. By taking LST in (3.6), we have (3.4). By using the same arguments as in Chapter 2 of Neuts [18] we have that $H^{*}(s)$ is the minimal nonnegative solution of

$$
H^{*}(s)=\left(\frac{\lambda_{1}+\lambda_{2}}{\lambda_{1}+\lambda_{2}+s}\right) \sum_{n=0}^{\infty} C_{n}\left[H^{*}(s)\right]^{n}
$$

Remarks: 1 . The $m \times m$ matrix $H^{*}(s)$ in (3.5) can be calculated recursively by setting $H_{0}^{*}(s)=O$ in

$$
H_{n+1}^{*}(s)=\left(\frac{\lambda_{1}+\lambda_{2}}{\lambda_{1}+\lambda_{2}+s}\right) \sum_{k=0}^{\infty} C_{k}\left[H_{n}^{*}(s)\right]^{k}
$$

For more details in calculation of $H^{*}(s)$, see Neuts [18].

2. $G_{Z}(-n, t)$ in Proposition 2 can be considered as a busy period distribution in

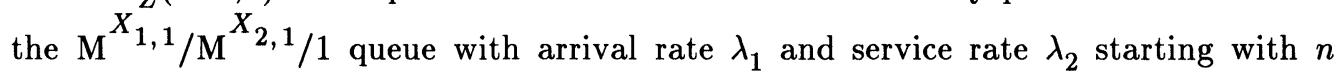
customers.

\section{Special Cases}

1. If $m>1$ and $l \leq m-1$, then $C_{n}=O, n \geq 2$ and hence we have

$$
H^{*}(s)=\left(\frac{\lambda_{1}+\lambda_{2}+s}{\lambda_{1}+\lambda_{2}} I-C_{1}\right)^{-1} C_{0},
$$

where $I$ is the $m \times m$ identity matrix.

2. If $m=1$, that is, $X_{2, i} \equiv 1$, then $G_{Z}^{*}(n, s)$ is obtained from (3.4) and (3.5) as

$$
G_{Z}^{*}(-n, s)=\left[H^{*}(s)\right]^{n}, n \geq 1
$$

and $H^{*}(s)$ is the solution of the equation

$$
z=\frac{1}{\lambda_{1}+\lambda_{2}+s}\left(\lambda_{2}+\lambda_{1} z \Xi_{1}(z)\right)
$$

with $|z|<1$, where $\Xi_{1}(z)=\sum_{i=1}^{\infty} x_{1, i} z^{i}$.

3. If $l=m=1$, that is, $X_{1, i} \equiv 1$ and $X_{2, i} \equiv 1$, then letting $\Xi_{1}(z)=z$ in (3.8) and solving equation (3.8), we have

$$
H^{*}(s)=\frac{1}{2 \lambda_{1}}\left(\left(\lambda_{1}+\lambda_{2}+s\right)=\sqrt{\left(\lambda_{1}+\lambda_{2}+s\right)^{2}-4 \lambda_{1} \lambda_{2}}\right) .
$$

\section{RCE With FCFS Discipline}

Under the FCFS queueing discipline with RCE removal strategy, upon arrival of a negative customer, if the number of positive customers is fewer than $B$, then all the 
positive customers are removed; otherwise, $B$ customers from the end of the queue are removed. Let $W$ denote the time period during which the tagged customer spends in the system from the epoch of arrival to the epoch of its service completion. We assume that $W$ is infinite if the tagged customer is removed from the system before its service completion. Let $N_{a}$ and $N_{b}$ be the numbers of customers ahead of and behind the tagged customer, respectively, immediately after its arrival instant, and let $N$ be the number of customers in the system at the tagged customer's arrival. Let $A^{*}$ and $A_{-}^{*}$ be the batch size to which the tagged customer belongs and the number of customers in the preceding batch. Note that the probability mass functions of $A^{*}$ and $A_{-}^{*}$ are given by

$$
P\left(A^{*}=k\right)=\frac{k a_{k}}{\bar{a}} \text { and } P\left(A_{-}^{*}=j \mid A^{*}=k\right)=\frac{1}{k}, j=0,1, \ldots, k-1 .
$$

Thus the distribution function $W(x)=P(W \leq x)$ in equilibrium is

$$
\begin{aligned}
W(x) & =\sum_{n=0}^{\infty} \pi_{n} P(W \leq x \mid N=n) \\
& =\sum_{n=0}^{\infty} \pi_{n} \sum_{k=1}^{l} P\left(W \leq x \mid N=n, A^{*}=k\right) \frac{k a_{k}}{\bar{a}} \\
& =\sum_{n=0}^{\infty} \pi_{n} \sum_{k=1}^{l} \frac{k a_{k}}{\bar{a}} \sum_{j=0}^{k-1} \frac{1}{k} P\left(W \leq x \mid N=n, A^{*}=k, A_{-}^{*}=j\right) \\
& =\sum_{n=0}^{\infty} \pi_{n} \sum_{k=1}^{l} \frac{a_{k}}{\bar{a}} \sum_{j=0}^{k-1} P\left(W \leq x \mid N_{a}=n+j, N_{b}=k-j-1\right) .
\end{aligned}
$$

To calculate the conditional distribution $P\left(W \leq x \mid N_{a}=n, N_{b}=k\right)$, we define the Markov chain

$$
X(t)=X^{+}(t)-X^{-}(t), t \geq 0
$$

with $X(0)=0$, where $X^{+}(t)$ and $X^{-}(t)$ are the numbers of positive customers having arrived and potential removals by negative customers up to time $t$, respectively. Then the $\operatorname{LST} G_{X}^{*}(-n, s)$ of the first passage time distribution function

$$
G_{X}(-n)=\inf \{t \geq 0: X(t) \leq-n\}, n \geq 1
$$

can be obtained from (3.4) by replacing $c_{i}$ in (3.3) by

$$
c_{i}=\left\{\begin{array}{cc}
\frac{\lambda^{+}}{\lambda} a_{i}, & \text { if } 1 \leq i \leq l \\
\frac{\lambda^{-}}{\lambda} b-i, & \text { if }-m \leq i \leq-1 \\
0, & \text { otherwise. }
\end{array}\right.
$$

Let $S_{n}$ be the time needed to serve $n$ consecutive customers. Since the service time distribution is exponential with parameter $\mu$, the probability density function $s_{n}(t)$ of $S_{n}$ is given by

$$
s_{n}(t)=\mu e^{-\mu t} \frac{(\mu t)^{n-1}}{(n-1) !}, t \geq 0
$$


Under the FCFS service discipline with RCE removal strategy, when $N_{a}=n, N_{b}=k$ for the tagged customer to complete its service without being removed, it must hold true that $G_{X}(-k-1)>S_{n+1}$. Hence, the conditional distribution of $W$ given that $N_{a}=n$ and $N_{b}=k$, is represented by

$$
\begin{aligned}
P\left(W \leq x \mid N_{a}\right. & \left.=n, N_{b}=k\right)=P\left(G_{X}(-k-1)>S_{n+1}, S_{n+1} \leq x\right) \\
& =\int_{0}^{x} P\left(G_{X}(-k-1)>t\right) s_{n+1}(t) d t .
\end{aligned}
$$

Letting

and

$$
\begin{gathered}
K_{j}(\alpha, s, t)=\sum_{n=0}^{\infty} \alpha^{n} e^{-s t} s_{n+j+1}(t) \\
=\alpha^{-j}\left[\mu e^{-(s+\mu(1-\alpha)) t}-\sum_{i=0}^{j-1} \frac{(\alpha \mu t)^{i}}{i !} \mu e^{-(s+\mu) t}\right], \quad j \geq 0,
\end{gathered}
$$

$$
\begin{gathered}
W(\alpha, x)=\sum_{n=0}^{\infty} \alpha^{n} P(W \leq x \mid N=n) \\
W^{*}(\alpha, s)=\int_{0}^{\infty} e^{-s x} W(\alpha, d x)
\end{gathered}
$$

we have from (2.1) and (4.1)-(4.3) the following proposition.

Proposition 3: The LST $W^{*}(s)$ of $W(x)$ is given by

where

$$
W^{*}(s)=C \sum_{i=1}^{K} \sum_{j=0}^{n_{i}-1} c_{i j}\left(\left.\frac{\partial^{j}}{\partial \alpha^{j}} W^{*}(\alpha, s)\right|_{\alpha=\alpha_{i}}\right)
$$

$$
\begin{aligned}
& W^{*}(\alpha, s)=\sum_{k=1}^{l} \frac{a_{k}}{\bar{a}} \sum_{j=0}^{k-1} \int_{0}^{\infty} K_{j}(\alpha, s, t)\left(1-G_{X}(j-k, t)\right) d t \\
& =\frac{\mu(s+\mu)\left(1-A\left(\frac{\mu}{s+\mu}\right)\right)}{\bar{a} s(s+\mu(1-\alpha))} \\
& -\frac{\mu}{s+\mu(1-\alpha)} \sum_{k=1}^{l} \frac{a_{k}}{\bar{a}} \sum_{j=0}^{k-1} \alpha^{-j} G_{X}^{*}(j-k, s+\mu(1-\alpha) \\
& +\sum_{k=1}^{l} \frac{a_{k}}{\bar{a}} \sum_{j=0}^{k-1} \alpha-j \sum_{i=0}^{j-1} \frac{(\alpha \mu)^{i}}{i !} \mu(-1)^{i} \frac{d^{i}}{d s^{i}}\left[\frac{G_{X}^{*}(j-k, s+\mu)}{s+\mu}\right] .
\end{aligned}
$$

\section{Special Cases}

1. If $l=1$, that is, $A \equiv 1$, then we have from (2.6), (3.4) and (4.5) that

$$
W^{*}(s)=\left(1-\alpha_{0}\right) W^{*}\left(\alpha_{0}, s\right)
$$




$$
\begin{aligned}
& =\frac{\mu\left(1-\alpha_{0}\right)}{s+\mu\left(1-\alpha_{0}\right)}\left[1-G_{X}^{*}\left(-1, s+\mu\left(1-\alpha_{0}\right)\right)\right] \\
= & \frac{\mu\left(1-\alpha_{0}\right)}{s+\mu\left(1-\alpha_{0}\right)}\left[1-\sum_{j=1}^{m}\left[H^{*}\left(s+\mu\left(1-\alpha_{0}\right)\right)\right]_{1, j}\right] .
\end{aligned}
$$

2. If $m=1$, that is, $B \equiv 1$, then $G_{X}^{*}(-n, s)=\left[H^{*}(s)\right]^{n}$ and (4.5) becomes

$$
\begin{gathered}
W^{*}(\alpha, s)=\frac{\mu(s+\mu)\left(1-A\left(\frac{\mu}{s+\mu}\right)\right)}{\bar{a} s(s+\mu(1-\alpha))} \\
-\frac{\mu}{s+\mu(1-\alpha)} \frac{\alpha \beta(\alpha, s)[A(\beta(\alpha, s))-A(1 / \alpha)]}{\bar{a}(\alpha \beta(\alpha, s)-1)} \\
+\sum_{k=1}^{l} \frac{a_{k}}{\bar{a}} \alpha-k \sum_{i=0}^{k-2} \frac{(\alpha \mu)^{i}}{i !} \mu(-1)^{i} \frac{d^{i}}{d s^{i}}\left[\frac{(\alpha \gamma(s))^{k-i}-\alpha \gamma(s)}{(s+\mu)(\alpha \gamma(s)-1)}\right]
\end{gathered}
$$

where $\beta(\alpha, s)=H^{*}(s+\mu(1-\alpha))$ and $\gamma(s)=H^{*}(s+\mu)$.

3. If $l=m=1$, then we have from (2.7) and (4.6) that

$$
W^{*}(s)=\frac{\mu(1-\rho)}{\mu(1-\rho)+s^{2}}\left[1-H^{*}(\mu(1-\rho)+s)\right]
$$

where $H^{*}(s)=\frac{1}{2 \lambda^{+}}\left[\lambda^{+}+\lambda^{-}+s-\sqrt{\left(\lambda^{+}+\lambda^{-}+s\right)^{2}-4 \lambda^{+} \lambda^{-}}\right]$.

Note that (4.8) coincides with the known result in Harrison and Pitel [11].

\section{RCH With the FCFS Discipline}

Under the RCH removal strategy, when a negative customer finds $n$ positive customers upon its arrival and $n \leq B$, then he removes all the positive customers. If $n>B$, then $B$ customers from the head containing the customer in service are removed, and hence the customers behind the tagged customer do not affect the sojourn time of the tagged customer. Thus, using the same argument as in (4.1), we have

$$
\begin{aligned}
P(W \leq x \mid N=n) & =\sum_{k=1}^{l} \frac{a_{k}}{\bar{a}} \sum_{j=0}^{k-1} P\left(W \leq x \mid N_{a}=n+j, N_{b}=k-j-1\right) \\
& =\sum_{k=1}^{l} \frac{a_{k}}{\bar{a}} \sum_{j=0}^{k-1} P\left(W \leq x \mid N_{a}=n+j\right)
\end{aligned}
$$

Letting $V_{n}(x)=P\left(W \leq x \mid N_{a}=n\right), n=0,1,2, \ldots$ and $V_{n}^{*}(s)$ be the LST of $V_{n}(x)$, the $\operatorname{LST} W^{*}(s)$ of $W(x)$ is given by

$$
W^{*}(s)=\sum_{n=0}^{\infty} \pi_{n} \sum_{k=1}^{l} \frac{a_{k}}{\bar{a}} \sum_{j=0}^{k-1} V_{n+j}^{*}(s) .
$$


Now we derive $V_{n}^{*}(s), n \geq 0$. Taking the conditional probability on the first departure of positive customers due to service completion or negative arrival and then using the total probability law, we have the following recursive relation:

$$
\begin{gathered}
V_{0}^{*}(s)=\frac{\mu}{\widetilde{\mu}} \xi(s), \\
V_{n}^{*}(s)=\xi(s)\left(\frac{\mu}{\widetilde{\mu}} V_{n-1}^{*}(s)+\frac{\lambda^{-}}{\widetilde{\mu}} \sum_{k=1}^{n} b_{k} V_{n-k}^{*}(s)\right), n \geq 1,
\end{gathered}
$$

where $\xi(s)=\frac{\tilde{\mu}}{\tilde{\mu}+s}$ and $b_{k}=0, k \geq n+1$. Simple calculation yields the generating function $V^{*}(\alpha, s)=\sum_{n=0}^{\infty} \alpha^{n} V_{n}^{*}(s)$ as

$$
V^{*}(\alpha, s)=\frac{\mu \xi(s)}{\tilde{\mu}-\left(\mu \alpha+\lambda^{-} B(\alpha)\right) \xi(s)}, \quad|\alpha| \leq 1, s>0
$$

We have from (2.1), (5.1) and (5.3) the following proposition.

Proposition 4: The LST $W^{*}(s)$ of $W(x)$ is given by

where

$$
W^{*}(s)=C \sum_{i=1}^{K} \sum_{j=0}^{n_{i}-1} c_{i j}\left(\left.\frac{\partial^{j}}{\partial \alpha^{j}} W^{*}(\alpha, s)\right|_{\alpha=\alpha_{i}}\right)
$$

$$
\begin{gathered}
W^{*}(\alpha, s)=\sum_{n=0}^{\infty} \alpha^{n} \sum_{k=1}^{l} \frac{a_{k}}{\bar{a}} \sum_{j=0}^{k-1} V_{n+j}^{*}(s) \\
=\sum_{k=1}^{l} \frac{a_{k}}{\bar{a}} \sum_{j=0}^{k-1} \alpha^{-j}\left[V^{*}(\alpha, s)-\sum_{i=0}^{j-1} \alpha^{i} V_{i}^{*}(s)\right] \\
=V^{*}(\alpha, s) \frac{1}{\bar{a}}\left(\frac{1-A\left(\frac{1}{\alpha}\right)}{1-\frac{1}{\alpha}}\right)-\sum_{k=2}^{l} \frac{a_{k}}{\bar{a}} \sum_{j=0}^{k-1} \sum_{i=0}^{j-1} \alpha^{i-j} V_{i}^{*}(s) .
\end{gathered}
$$

\section{Special Cases}

1. If $A \equiv 1$, then we have from (5.5) that $W^{*}(\alpha, s)=V^{*}(\alpha, s)$ and hence, from (2.6) and (5.3) that

$$
W^{*}(s)=\frac{\left(1-\alpha_{0}\right) \mu \xi(s)}{\tilde{\mu}-\left(\mu \alpha_{0}+\lambda^{-} B\left(\alpha_{0}\right)\right) \xi(s)}
$$

2. If $B \equiv 1$, then it is easy to see from (5.3) that

$$
V^{*}(\alpha, s)=\frac{\mu}{\widetilde{\mu}} \xi(s) \frac{1}{1-\xi(s) \alpha}=\sum_{n=0}^{\infty} \frac{\mu}{\widetilde{\mu}} \xi(s)^{n+1} \alpha^{n}
$$

and hence $V_{n}^{*}(s)=\frac{\mu}{\widetilde{\mu}} \xi(s)^{n+1}, n \geq 0$. Thus we have

$$
W^{*}(\alpha, s)=\left(\frac{\mu}{\bar{a} \widetilde{\mu}}\right)\left(\frac{\xi(s)}{1-\alpha \xi(s)}\right)\left(\frac{1-A(\xi(s))}{1-\xi(s)}\right)
$$


3. If $A \equiv 1$ and $B \equiv 1$, then since $\alpha_{0}=\rho$ and $B(\alpha)=\alpha$, we have from (5.6) that

$$
W^{*}(s)=\left(\frac{\mu}{\tilde{\mu}}\right)\left(\frac{\tilde{\mu}-\lambda^{+}}{\tilde{\mu}-\lambda^{+}+s}\right)
$$

which is identical to the formula in Harrison and Pitel [11].

\section{RCE With Preemptive LCFS Discipline}

Here, the batch to which the tagged customer belongs goes immediately to the front of the queue and the removal of customers by a negative arrival is done at the end of the queue. Thus, when $N_{a}=n, N_{b}=k$, for the tagged customer to complete its service without being removed, the tagged customer must complete its service before the negative arrivals remove $k+1$ positive customers. Note that when $N_{a}=n$, the time period that the tagged customer completes its service is the same as the busy period $\Psi_{n+1}$ in an ordinary $\mathrm{M}^{A} / \mathrm{M} / 1$ queue starting with $n+1$ customers. Let $X^{-}(t)$ be the numbers of potential removals by negative customers up to time $t$ and

$$
U_{X^{-}}(n)=\inf \left\{t \geq 0, X^{-}(t) \geq n\right\}
$$

and $\Psi(n, t)=P\left(\Psi_{n} \leq t\right)$. Then we have

$$
\begin{aligned}
P\left(W \leq x \mid N_{a}\right. & \left.=n, N_{b}=k\right)=P\left(U_{X}-(k+1)>\Psi_{n+1}, \Psi_{n+1} \leq x\right) \\
& =\int_{0}^{x} P\left(U_{X}-(k+1)>t\right) \Psi(n+1, d t) .
\end{aligned}
$$

The double transform $\tilde{U}_{X}^{*}-(z, s)$ of $U_{X}-(n, x)=P\left(U_{X}-(n) \leq x\right)$ is obtained from (3.2) as

$$
\widetilde{U}_{X}-(z, s)=\frac{z}{1-z} \frac{\lambda^{-}(1-B(z))}{s+\lambda^{-}(1-B(z))}
$$

Denoting by $\Psi^{*}(n, s)$ the LST of $\Psi(n, t)$ and $\Psi^{*}(s) \equiv \Psi^{*}(1, s)$, we have from $(3.7)$ and (3.8) that

$$
\Psi^{*}(n, s)=\left[\Psi^{*}(s)\right]^{n}
$$

where $\Psi^{*}(s)$ is the unique solution of the equation

$$
z=\frac{\mu+\lambda^{+} z A(z)}{s+\mu+\lambda^{+}}
$$

with $|z|<1$. Following the same procedure as (4.1), we have from (6.1) that

$$
W(x)=\sum_{n=0}^{\infty} \pi_{n} \sum_{k=1}^{l} \frac{a_{k}}{\bar{a}} \sum_{i=0}^{k-1} \int_{0}^{x}\left[1-U_{X}-(n+k-i, t)\right] \Psi(i+1, d t) .
$$

Now, we have from (2.1) and (6.2) the following proposition. 
Proposition 5: The $\operatorname{LST} W^{*}(s)$ of $W(x)$ is given by

where

$$
W^{*}(s)=C \sum_{i=1}^{K} \sum_{j=0}^{n_{i}-1} c_{i j}\left(\left.\frac{\partial^{j}}{\partial \alpha^{j}} W^{*}(\alpha, s)\right|_{\alpha=\alpha_{i}}\right)
$$

$$
\begin{gathered}
W^{*}(\alpha, s)=\frac{1}{1-\alpha} \frac{\Psi^{*}(s)\left[1-A\left(\Psi^{*}(s)\right)\right]}{\bar{a}\left(1-\Psi^{*}(s)\right)} \\
-\sum_{n=0}^{\infty} \alpha^{n} \sum_{k=1}^{l} \frac{a_{k}}{\bar{a}} \sum_{i=0}^{k-1} \int_{0}^{\infty} e^{-s t} U_{X}-(n+k-i, t) \Psi(i+1, d t) .
\end{gathered}
$$

\section{Special Cases}

1. If $A \equiv 1$, then we have

$$
\begin{gathered}
W^{*}(s)=\left(1-\alpha_{0}\right) W^{*}\left(\alpha_{0}, s\right) \\
=\Psi^{*}(s)-\frac{1-\alpha_{0}}{\alpha_{0}} \int_{0}^{\infty} e^{-s t} \widetilde{U}_{X}-\left(\alpha_{0}, t\right) \Psi(d t),
\end{gathered}
$$

where $\tilde{U}_{X}-(z, t)=\sum_{n=1}^{\infty} z^{n} U_{X}-(n, t)$ and

$$
\Psi^{*}(s)=\frac{1}{2 \lambda^{+}}\left(\lambda^{+}+\mu+s-\sqrt{\left(\lambda^{+}+\mu+s\right)^{2}-4 \lambda^{+} \mu}\right) .
$$

2. If $B \equiv 1$, then

$$
U_{X}-(n, t)=1-\sum_{j=0}^{n-1} e^{-\lambda-} \frac{\left(\lambda^{-} t\right)^{j}}{j !}, n \geq 1,
$$

and hence we have

$$
\begin{gathered}
W^{*}(\alpha, s)=\sum_{n=0}^{\infty} \alpha^{n} \sum_{k=1}^{l} \frac{a_{k}}{\bar{a}} \sum_{i=0}^{k-1} \sum_{j=0}^{n+k-i-1} \frac{\left(\lambda^{-}\right)^{j}}{j !} \int_{0}^{\infty} e^{-\left(s+\lambda^{-}\right) t} t^{j} \Psi(i+1, d t) \\
=\sum_{n=0}^{\infty} \alpha^{n} \sum_{k=1}^{l} \frac{a_{k}}{\bar{a}} \sum_{i=0}^{k-1} \sum_{j=0}^{n+k-i-1} \frac{\left(\lambda^{-}\right)^{j}}{j !}(-1)^{j} \Psi^{*(j)}\left(i+1, \lambda^{-}+s\right), \\
\quad \text { where } \Psi^{*(j)}(n, s)=\frac{d^{j}}{d s^{j}} \Psi^{*}(n, s) .
\end{gathered}
$$

3. If $A \equiv 1$ and $B \equiv 1$, then we have from (6.6) that

$$
W^{*}(\alpha, s)=\frac{1}{1-\alpha} \Psi^{*}\left(s+\lambda^{-}(1-\alpha)\right)
$$

and hence from (2.7) that 


$$
\begin{gathered}
W^{*}(s)=\Psi^{*}\left(s+\lambda^{-}(1-\rho)\right) \\
=\frac{1}{2 \lambda^{+}}\left(s+\lambda+\mu-\lambda^{-} \rho-\sqrt{\left(s+\lambda+\mu-\lambda^{-} \rho\right)^{2}-4 \lambda^{+} \mu}\right),
\end{gathered}
$$

which is the same as that in Harrison and Pitel [11].

\section{RCH With Preemptive LCFS Discipline}

Let $\left\{X^{+}(t), t \geq 0\right\}$ and $\{Y(t), t \geq 0\}$ be independent compound Poisson processes with their one-dimensional distributions as those of the number of the arrived positive customers and the number of positive customers potentially leaving the system due to service completion or negative arrivals up to time $t$, respectively. We define a Markov chain $\{Z(t), t \geq 0\}$ by

$$
Z(t)=X^{+}(t)-Y(t)
$$

Here the queue left behind the tagged customer is irrelevant throughout its sojourn time. Thus, using the same argument as (4.1), we have

$$
W(x)=\sum_{k=1}^{l} \frac{a_{k}}{\bar{a}} \sum_{j=0}^{k-1} P\left(W \leq x \mid N_{a}=j\right) .
$$

Define $V_{n}(x)=P\left(W \leq x \mid N_{a}=n\right)$ and denote its LST by $V_{n}^{*}(s)$. For the notational simplicity, we let

$$
\begin{gathered}
\eta(s)=\frac{\lambda^{+}+\tilde{\mu}}{\lambda^{+}+\tilde{\mu}+s}, \\
\omega=\frac{\mu}{\lambda^{+}+\tilde{\mu}}, \\
q_{k}=\left\{\begin{array}{cc}
\frac{\lambda^{+} a_{k}}{\lambda^{+}+\tilde{\mu}}, & \text { if } 1 \leq k \leq l \\
0, & \text { otherwise, }
\end{array}\right. \\
r_{k}=\left\{\begin{array}{cc}
\frac{\mu+\lambda^{-} b_{1}}{\lambda^{+}+\tilde{\mu}}, & \text { if } k=1 \\
\frac{\lambda^{-} b_{k}}{\lambda^{+}+\tilde{\mu}}, & \text { if } k \geq 1 \\
0, & \text { otherwise. }
\end{array}\right.
\end{gathered}
$$

Taking the conditional probability on an arrival of positive customers or departure of positive customers due to service completion or negative arrival and then applying the total probability law, we have the following relations:

$$
V_{0}^{*}(s)=\eta(s)\left(\omega+\sum_{k=1}^{l} q_{k} V_{k}^{*}(s)\right)
$$




$$
V_{n}^{*}(s)=\eta(s)\left(\sum_{k=1}^{\min (m, n)} r_{k} V_{n-k}^{*}(s)+\sum_{k=1}^{l} q_{k} V_{n+k}^{*}(s)\right), n \geq 1
$$

Let $\left[H^{[\nu] *}(s)\right]_{i j}(\nu=1,2, \ldots, 1 \leq i, j \leq m)$ be the LST of the distribution function of the first hitting time of $\{Z(t), t \geq 0\}$ from state $m \nu+i-1$ to state $j-1$ and $H^{[\nu] *}(s)$ the $m \times m$ matrix with $(i, j)$-entry $\left[H^{[\nu] *}(s)\right]_{i j}$. Following the same arguments as in Proposition 2, we have that

$$
H^{[\nu] *}(s)=\left[H^{*}(s)\right]^{\nu}, \quad \nu=1,2, \ldots,
$$

where $m \times m$ matrix $H^{*}(s)$ is the minimal nonnegative solution of the matrix equation

$$
H^{*}(s)=\eta(s) \sum_{n=0}^{\infty} C_{n}\left[H^{*}(s)\right]^{n}
$$

where the matrices $C_{n}$ 's are of the same form as those in Section 3 with

$$
c_{k}=\left\{\begin{array}{cc}
q_{k}, & \text { if } 1 \leq k \leq l \\
r_{k}, & \text { if }-m \leq k \leq-1 \\
0, & \text { otherwise. }
\end{array}\right.
$$

Let $\boldsymbol{V}^{*}(s, n)=\left(V_{m n}^{*}(s), V_{m n+1}^{*}(s), \ldots, V_{m(n+1)-1}^{*}(s)\right)^{t}, n=0,1,2, \ldots$ be $m$-vectors. Then it is easy to see that

$$
\boldsymbol{V}^{*}(s, n)=\left[H^{*}(s)\right]^{n} \boldsymbol{V}^{*}(s, 0), n=0,1,2, \ldots,
$$

with $\left[H^{*}(s)\right]^{0}=I$ being the $m \times m$ identity matrix. Rewriting (7.2) and using (7.4) yield

$$
\begin{gathered}
V_{0}^{*}(s)=\eta(s) \omega+\eta(s) \sum_{k=0}^{\infty} \gamma_{0}(k)\left[H^{*}(s)\right]^{k} \boldsymbol{V}^{*}(s, 0) \\
V_{n}^{*}(s)=\eta(s) \sum_{k=0}^{\infty} \boldsymbol{\gamma}_{n}(k)\left[H^{*}(s)\right]^{k} \boldsymbol{V}^{*}(s, 0), 1 \leq n \leq m-1,
\end{gathered}
$$

where

$$
\gamma_{n}(k)=\left(c_{m k-n}, c_{m k-n+1}, \ldots, c_{m(k+1)-n-1}\right), 0 \leq n \leq m-1, k \geq 0 .
$$

Hence we get the following matrix form representation of $\boldsymbol{V}^{*}(s, 0)$ :

$$
\boldsymbol{V}^{*}(s, 0)=\left[I-\eta(s) \sum_{k=0}^{\infty} \Gamma_{k}\left[H^{*}(s)\right]^{k}\right]^{-1} \eta(s) \omega \boldsymbol{e}_{1},
$$

where $\Gamma_{k}$ is the $m \times m$ matrix whose $j$ th row is $\gamma_{j}(k), 0 \leq j \leq m-1$ and $e_{1}=$ $(1,0, \ldots, 0)^{t}$ is $m$-vector. The existence of the matrix inversion in (7.6) is obvious, since the matrix is strictly diagonally dominant. The infinite sum of matrices in (7.6) is in fact a finite sum, since $\Gamma_{k}=O$ for $k \geq \nu+2$, where $m \nu \leq l<m(\nu+1)$.

Proposition 6: The $L S T W^{*}(s)$ of $W(x)$ is given by 


$$
W^{*}(s)=\sum_{k=1}^{l} \frac{a_{k}}{\bar{a}} \sum_{j=0}^{k-1} V_{j}^{*}(s)
$$

where $V_{n}^{*}(s), 0 \leq n \leq l-1$ are obtained from (7.4) and (7.6).

\section{Special Cases}

1. If $A \equiv 1$, then we have from (7.7) that

and

$$
W^{*}(s)=V_{0}^{*}(s)
$$

$$
\boldsymbol{V}^{*}(s, 0)=\left[I-\eta(s)\left(\Gamma_{0}+\Gamma_{1} H^{*}(s)\right)\right]^{-1} \eta(s) \omega \boldsymbol{e}_{1},
$$

where

$$
\Gamma_{0}=\left(\begin{array}{ccccc}
0 & q_{1} & 0 & \ldots & 0 \\
r_{1} & 0 & q_{1} & \ldots & 0 \\
\vdots & \ddots & \ddots & \ddots & \vdots \\
r_{m-2} & \ldots & r_{1} & 0 & q_{1} \\
r_{m-1} & r_{m-2} & \ldots & r_{1} & 0
\end{array}\right), \Gamma_{1}=\left(\begin{array}{l} 
\\
\\
\end{array}\right)
$$

2. If $B \equiv 1$, then we have from (3.8) that $H^{*}(s)$ is the unique solution of the equation

$$
z=\frac{1}{\lambda^{+}+\tilde{\mu}+s}\left(\tilde{\mu}+\lambda^{+} z A(z)\right)
$$

with $|z|<1$. (7.6) leads to

$$
V_{0}^{*}(s)=\frac{\eta(s) \omega}{1-\frac{\lambda^{+}}{\lambda^{+}+\widetilde{\mu}+s} A\left(H^{*}(s)\right)}=\frac{\mu}{\widetilde{\mu}} H^{*}(s) .
$$

Thus,

$$
\begin{gathered}
W^{*}(s)=\sum_{k=1}^{l} \frac{a_{k}}{\bar{a}} V_{0}^{*}(s) \frac{1-\left[H^{*}(s)\right]^{k}}{1-H^{*}(s)} \\
=\frac{\mu}{\tilde{\mu}} \frac{H^{*}(s)\left(1-A\left(H^{*}(s)\right)\right)}{\bar{a}\left(1-H^{*}(s)\right)} .
\end{gathered}
$$

3. If $A \equiv 1$ and $B \equiv 1$, then by letting $A(z)=z$ in (7.9) we have from (3.9) that

$$
W^{*}(s)=\frac{\mu}{\tilde{\mu}} \frac{1}{2 \lambda^{+}}\left(\left(\lambda^{+}+\tilde{\mu}+s\right)-\sqrt{\left.\left(\lambda^{+}+\tilde{\mu}+s\right)^{2}-r \lambda^{+} \tilde{\mu}\right)}\right)
$$

which is the same as that in Harrison and Pitel [11].

\section{Acknowledgement}

The author wishes to acknowledge the financial support of the Korean Research Foun- 
dation made in the program year of 1997. The author is grateful to the anonymous referee for his/her suggestions that improved the presentation of the paper.

\section{References}

[1] Bayer, N. and Boxma, O.J., Wiener-Hopf analysis of an M/G/1 queue with negative customers and of a related class of random walks, Queueing Systems $\mathbf{2 3}$ (1996), 301-316.

[2] Boucherie, R.J. and Boxma, O.J., The workload in the M/G/1 queue with work removal, Prob. Engin. Info. Sci. 10 (1996), 261-277.

[3] Boucherie, R.J. and van Dijk, N.M., Local balance in queueing networks with positive and negative customers, Ann. Oper. Res. 48 (1994), 463-492.

[4] Chao, X., A queueing network model with catastrophes and product form solution, Oper. Res. Letters 18 (1995), 75-79.

[5] Chao, X. and Pinedo, M., Generalized networks of queues with positive and negative arrivals, Prob. Engin. Info. Sci. 7 (1993), 301-334.

[6] Fourneau, J.M., Gelenbe, E. and Suros, R., G-networks with multiple classes of negative and positive customers, Theoret. Comput. Sci. 155 (1996), 141-156.

[7] Gelenbe, E., Product form network with negative and positive customers, $J$. Appl. Prob. 28 (1991), 656-663..

[8] Gelenbe, E., G-networks with triggered customer movement, J. Appl. Prob. 30 (1993), 742-748.

[9] Gelenbe, E., G-networks: a unifying model for neural and queueing networks, Ann. Oper. Res. 48 (1994), 433-461.

[10] Gelenbe, E., Glynn, P.G. and Sigman, K., Queues with negative arrivals, J. Appl. Prob. 28 (1991), 245-250.

[11] Harrison, P.G. and Pitel, E., Sojourn times in single server queues with negative customers, J. Appl. Prob. 30 (1993), 943-963.

[12] Harrison, P.G. and Pitel, E., Response time distributions in tandem $G$-networks, J. Appl. Prob. 32 (1995), 224-246.

[13] Harrison, P.G. and Pitel, E., The M/G/1 queue with negative customers, $A d v$. Appl. Prob. 28 (1996), 540-556.

[14] Henderson, W., Queueing networks with negative customers and negative queue lengths, J. Appl. Prob. 30:4 (1993), 931-942.

[15] Henderson, W., Northcote, B.S. and Taylor, P.G., Geometric equilibrium distribution for queue with interactive batch departures, Ann. Oper. Res. 48 (1994), 493-511.

[16] Jain, G. and Sigman, K.A., Pollaczek-Khinchine formulation for M/G/1 queues with disasters, J. Appl. Prob. 33 (1996), 1191-1200.

[17] Miller, R.G., A contribution to the theory of bulk queues, J. Royal Stat. Soc. Ser. B21 (1959), 320-337.

[18] Neuts, M.F., Structured Stochastic Matrices of M/G/1 Type and Their Applications, Marcel Dekker, New York 1989. 


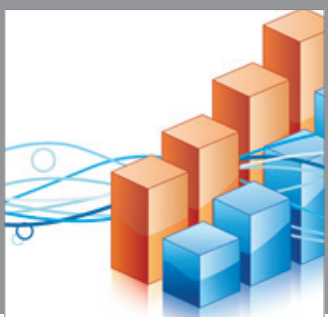

Advances in

Operations Research

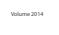

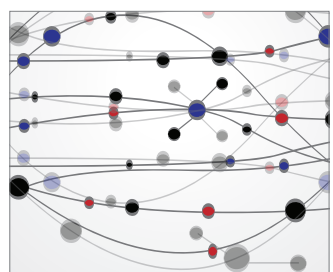

\section{The Scientific} World Journal
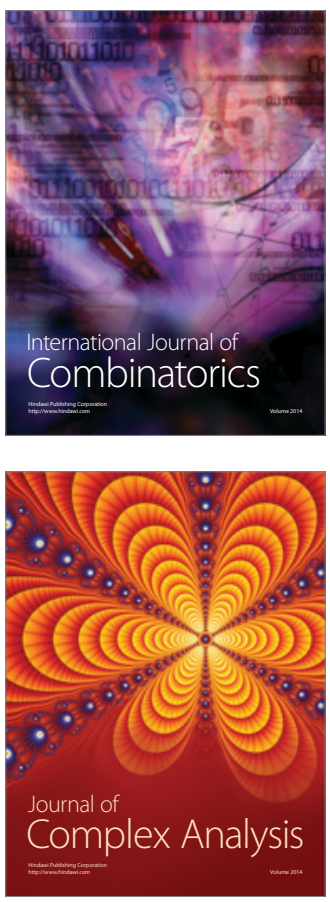

International Journal of

Mathematics and

Mathematical

Sciences
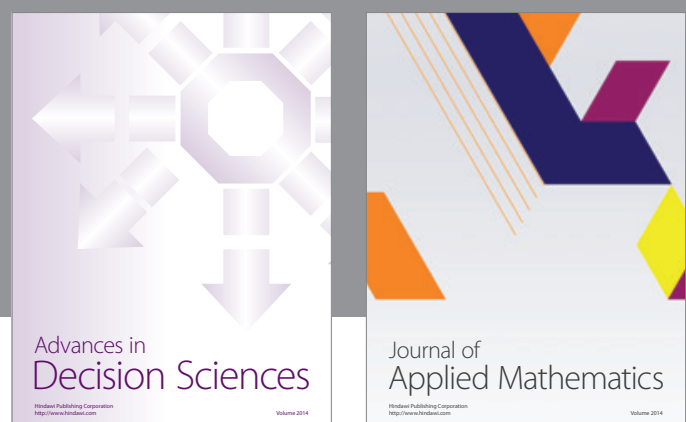

Journal of

Applied Mathematics
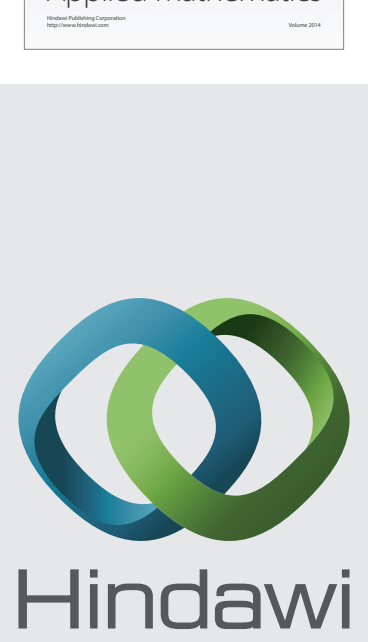

Submit your manuscripts at http://www.hindawi.com
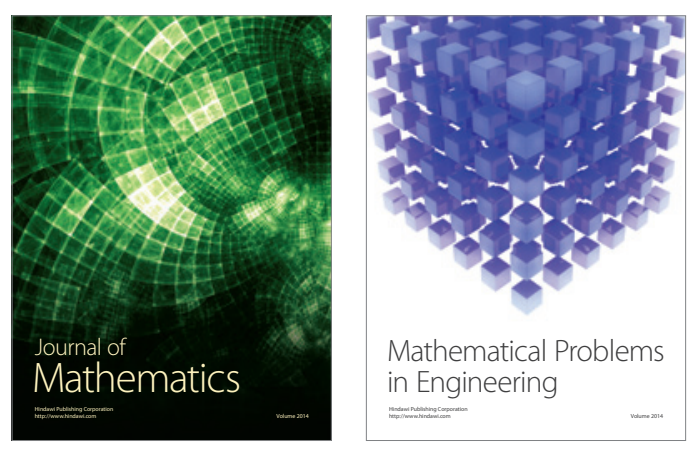

Mathematical Problems in Engineering
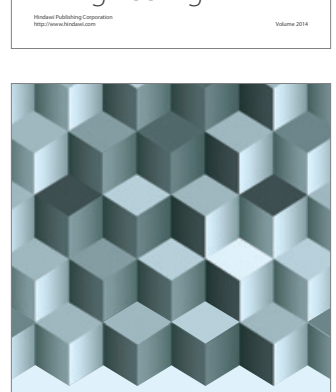

Journal of

Function Spaces
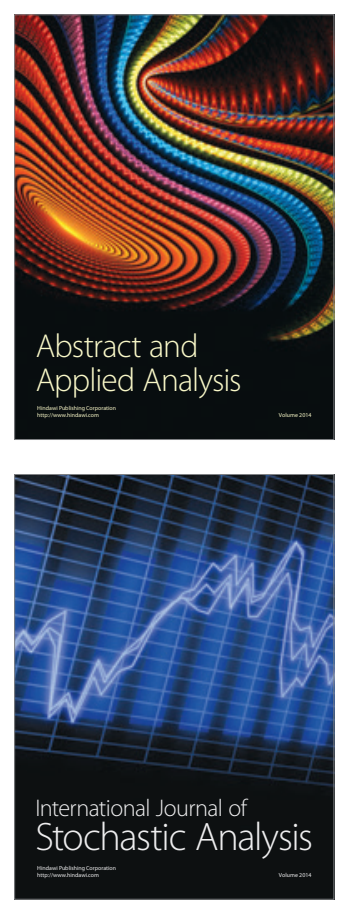

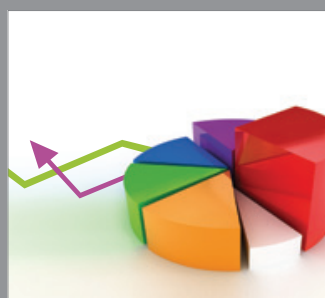

ournal of

Probability and Statistics

Promensencen
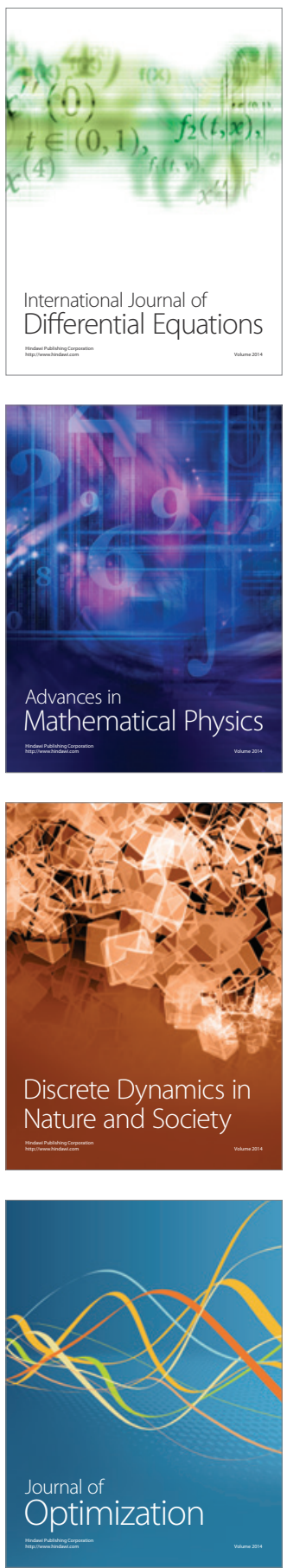